\title{
3D Ultrasound-CT Registration in Orthopaedic Trauma Using GMM Registration with Optimized Particle Simulation-Based Data Reduction
}

\author{
Ilker Hacihaliloglu ${ }^{1}$, Anna Brounstein ${ }^{2}$, Pierre Guy ${ }^{1}$, \\ Antony Hodgson ${ }^{3}$, and Rafeef Abugharbieh ${ }^{2}$ \\ ${ }^{1}$ Departments of Orthopaedics, \\ ${ }^{2}$ Departments of Electrical and Computer Engineering, \\ ${ }^{3}$ Mechanical Engineering, \\ University of British Columbia, Vancouver, BC, Canada \\ ilker.hacihaliloglu@hiphealth.ca, \{abrou, rafeef\}@ece.ubc.ca, \\ pierre.guy@ubc.ca, ahodgson@mech.ubc.ca
}

\begin{abstract}
Accurate real-time registration of intra-operative ultrasound (US) to computed tomography (CT) remains a challenging problem. In orthopedic applications, a recent promising approach proposed the use of Gaussian mixture modeling for bone surface registration. Though relatively successful, the method relied on naïve and error prone subsampling of the surfaces registered to reduce computational cost and also heavily relied on heuristically-set parameters for bone surface generation. In this paper, we present an improved approach employing a novel point simplification method that redistributes surface points to better represent the surface achieving near real-time registration with higher accuracy and robustness. We also present a framework for automating the parameter selection in the bone surface extraction step. For validation, we present extensive quantitative tests on phantom and clinical data obtained by scanning patients with pelvic ring fractures in the operating room. We show an $89 \%$ average improvement in target registration error over the recent GMM registration based method.
\end{abstract}

Keywords: intra-operative volume registration, 3D ultrasound to CT registration, orhopaedic imaging, real-time registration.

\section{Introduction}

Registering tracked intra-operative US images with pre-operative CT data has been proposed as a mechanism for making pre-operative CT more readily available for a range of computer assisted orthopaedic surgery (CAOS) procedures. The most widely used registration method in CAOS applications is the iterative closest point (ICP) algorithm, but since the initial publication of the ICP, new methods and algorithms have been proposed to improve the robustness and speed of the standard ICP algorithm [1]. Although relatively successful, ICP is susceptible to converging to local minima and therefore a close initial manual alignment is necessary. Moghari [2] proposed a point-based registration method based on the Unscented Kalman Filter 
(UKF). Although successful registration results were achieved, with a mean registration error of $0.3 \mathrm{~mm}$, the registration time exceeded 20 seconds due to the higher computational complexity of the method. The bone surfaces were also extracted manually from US images before the registration process, which would preclude direct use of this technique intraoperatively. Penney et al. [3] used a normalized cross-correlation similarity metric to register bone probability images obtained from CT and US data sets. They reported a mean target registration error (TRE) of $2.3 \mathrm{~mm}$ for cadaver study where the registration time was between 2 and $10.5 \mathrm{~min}$, which is well over the time needed for providing real-time guidance. Gill et al. [4] simulated US images from CT data for registering bone surfaces of the spine and achieved a registration accuracy of $1.44 \mathrm{~mm}$ for phantom scans and $1.25 \mathrm{~mm}$ for sheep cadaver scans. However their intensity-based registration took an average of 14 minutes on a central processing unit (CPU) and 11 seconds when implemented on a graphics processing unit (GPU). This approach was later extended to register statistical shape models (SSMs) of the lumbar spine where a TRE less than $3 \mathrm{~mm}$ for phantom scans was reported [5]. Again, the registration time was on the order of hours. Recently, Brounstein et al. [6] proposed a Gaussian Mixture Model-based (GMM) surface registration algorithm. The bone surfaces were automatically extracted using local phase image features obtained by convolving the US volumes with 3D Log-Gabor filters where the filter parameters were selected empirically [7]. In order to improve the speed of the proposed algorithm, the extracted point clouds were reduced using a simple downsampling approach that kept only $5 \%$ of the surface points [6]. The proposed method was evaluated on a phantom setup and three clinical scans. Although the early results were promising, some significant problems remained. For example, while local phase-based techniques successfully extract the desired image features, they remain sensitive to the underlying filter parameters used. Furthermore, the registration could fail if key features were lost in the down sampling process.

In this work, we propose and test several improvements to this work, including (1) extracting bone surfaces using automatically optimized 3D Log-Gabor filter parameters, (2) using a novel point cloud simplification method that ensures fast run time and retains salient features needed to provide more robust and accurate registration results; and (3) introducing an optimization method that reduces the complexity of point simplification by $50 \%$. We present validation studies not only on phantom data but also on an extensive set of clinical scans obtained from 21 human subjects with pelvic fractures to assess registration accuracy and robustness in the presence of typical US imaging artifacts.

\section{Methods}

\subsection{US-CT Registration Using Gaussian Mixture Models (GMMs)}

Gaussian Mixture Models (GMMs) are a way to replace a set of points in a multidimensional space with a (typically smaller) set of multi-dimensional Gaussian distributions (components) to describe sub-populations. Each component's density, $\varphi_{i}$, is characterized by its mean, $\mu_{i}$, and its covariance matrix, $\Sigma_{i}$. By representing the 
point sets as GMMs, we can compute an L2 distance metric between two different GMMs without the need to solve a computationally challenging point-to-point correspondence problem, which is normally required in many registration methods. The registration algorithm then minimizes this L2 distance between the model, $M(x)=$ $p_{m}(x)$, and the scene, $S(x)=p_{s}(x)$ over the set of possible rigid transformations, $T(M(x), \theta, t)$, where $\theta$ is a 6 -vector representing a rigid transform and the $L 2$ distance is given as:

$$
d_{L 2}(M(x), S(x), \theta, t)=\int(S(x)-T(M(x), \theta, t))^{2} d x
$$

It should be noted that the scene model, $S(x)$, is fixed during the optimization. Since $\mathrm{T}(\mathrm{M}(\mathrm{x}), \theta, \mathrm{t})$ and $\int T(M(x), \theta, t)^{2} d x$ are invariant for rigid transformations, minimizing the L2 distance given in Equation 1 becomes equivalent to solving:

$$
\operatorname{argmin}_{\theta, t}\left[d_{L 2}(M(x), S(x), \theta, t)\right]=\operatorname{argmin}_{\theta, t}\left[-\int S(x) T(M(x), \theta, t) d x\right]
$$

where $\int \varnothing\left(\boldsymbol{x} \mid \mu_{1}, \sum_{1}\right) \emptyset\left(\boldsymbol{x} \mid \mu_{2}, \sum_{2}\right) d x=\emptyset\left(0 \mid \mu_{1}-\mu_{2}, \sum_{1}+\sum_{2}\right)$. A closed form expression for the L2 distance between GMMs can be found in [8]. In [6], this approach was used for registering CT scans to local phase bone surfaces extracted from US volumes using 3D Log-Gabor filters with empirical filter parameters. The CT volumes were segmented using a binary threshold at 200 H.U [6]. Ray-casting was then used to find the bone surface in these binary CT volumes resulting in extracted surfaces that are one voxel thick. In order to achieve real-time registration the extracted bone surfaces were reduced using a simple/naïve down sampling approach by keeping only $5 \%$ of the total surface points. Finally, Gaussian curvature information was incorporated back into the reduced point clouds to provide correct registration along high curvature bone surface areas [6]. A critical consideration for successful local phase based feature extraction in medical images is the proper configuration of the different and highly sensitive parameters involved. Parameter values typically need to be scene-adaptive in order to accurately localize structures of interest in the scanned areas. This is especially important for extracting bone surfaces in US since in clinical scenarios the response of soft tissue interfaces can often resemble that of the bone. Furthermore, naïve down sampling can be problematic as it may not capture all the anatomical features the bone surface can offer, nor provide an even spread in the point cloud. Furthermore, it will allow outliers to influence the shape of the point cloud more and it is even possible for entire planes of the bone surface to be omitted, requiring more points in each point cloud to represent the surface which dramatically increases the runtime. In the next sections we provide solutions to these problems by proposing a framework for automatic point cloud optimization.

\subsection{Bone Surface Extraction Using Optimized 3D Log-Gabor Filters}

The use of 3D phase symmetry (3DPS) to automatically extract bone surfaces in US based on Log-Gabor filters was originally proposed in [7]. The transfer function of a 3D Log-Gabor filter, $3 D G_{i j}$, in the frequency domain can be constructed as the product of two components (3); a one dimensional Log Gabor function controlling the 
frequencies to which the filter responds, and a rotational symmetric angular Gaussian function that controls the orientation selectivity of the filter [7]:

$$
3 D G_{i j}=\exp \left(-\frac{\log ^{2}\left(\omega / \omega_{0 i}\right)}{2 \times \log ^{2}\left(\kappa / \omega_{0 i}\right)}\right) \times \exp \left(-\frac{\alpha\left(\phi_{j}, \theta_{j}\right)^{2}}{2 \times \sigma_{\alpha}^{2}}\right)
$$

The subscripts $i$ and $j$ denote the number of scale and orientations of the filter, respectively. $\alpha\left(\phi_{j}, \theta_{j}\right)$ is the angle between the azimuth $\left(\phi_{j}\right)$ and elevation $\left(\theta_{j}\right)$ angles. The angular bandwidth is determined by $\sigma_{\alpha} . \kappa$ is a scaling factor used to set the bandwidth of the filter in the radial direction. The scaling of the radial Log-Gabor function is controlled using different wavelengths that are based on multiples of a minimum wavelength, $\lambda_{\min }$, a user-defined parameter [7]. The filter scale $i$, and center frequency $\omega_{0}$ are related as $\omega_{0 i}=2 / \lambda_{\min } \times(\delta)^{\mathrm{i}-1}$ where $\delta$ is a scaling factor defined for computing the center frequencies of successive filters. In [9], Hacihaliloglu et al. proposed a framework for automating the selection of 2D Log-Gabor filter parameters. However, 2D methods ignore the correlations between adjacent slices and are therefore subject to large spatial compounding errors as well as errors associated with the US beam thickness effects [7]. Therefore, we have extended the automated filter parameter selection approach from slice-based 2D processing to fully 3D.Using this statistical approach we automatically selected all these filter parameters: frequency bandwidth $\left(\kappa / \omega_{0}\right)$, orientation (azimuth $\left(\phi_{j}\right)$ and elevation $\left(\theta_{j}\right)$ angles), scale $\left(\lambda_{\min }\right)$ and angular bandwidth $\left(\sigma_{\alpha}\right)$. The filter bandwidth in the radial direction, $\beta=-2 \times(2 / \ln 2)^{0.5} \times \ln \left(\kappa / \omega_{0}\right)$, is related to both the speckle and boundary responses in the US image. To determine the filter frequency bandwidth we analyzed US images with fully developed speckle in elevation direction. The speckle size is estimated as the full-width at half-maximum $(F W H M)$ of the autocorrelations. The ratio, $\kappa / \omega_{0}$, is computed as $\kappa / \omega_{0}=\exp (-0.25 \times$ $\left.(2 \times \ln (2))^{0.5} \times F W H M \times r\right)$. Here $r$ is the pixel size in $\mathrm{mm}$. The strongest response occurs when the 3D Log-Gabor filter is orientated orthogonally to the surface of the bone. The initial filter angles are obtained by clustering the 3D radon transform $(3 D R T)$ of the $3 \mathrm{D}$ B-mode US volume. The projection angles corresponding to the peak values of the $3 D R T$ represent the angles perpendicular to the high intensity bone response and are chosen as the initial filter angles. These initial angles are used to determine the filter scale $\left(\lambda_{\text {min }}\right)$. Since our main interest here is localizing bone contours, which generally appear as ridges in US images, we employ a metric that captures the 'ridgness' which we calculate as: $3 D R S_{\gamma}=t^{2 \gamma} \times\left(\mathrm{K}_{1}-\mathrm{K}_{2}\right)^{2}$. Here $t$ is the scale of the filter $\left(t=\lambda_{\text {min }}\right), \mathrm{K}_{1}=\operatorname{Trace}(H(x))+\left(\operatorname{Trace}(H(x))^{2}-4 \times \operatorname{det}(H(x))\right)^{0.5}$, and $K_{2}=\operatorname{Trace}(H(x))$ $\left.\operatorname{Trace}(H(x))^{2}-4 \times \operatorname{det}(H(x))\right)^{0.5} . H$ is the 3D Hessian matrix where 3D Log-Gabor filter was used during the construction of matrix. We analyze the intensity distribution of $3 D R S_{\gamma}$ over all possible scales and select the scale where the sum of intensities is maximized as the correct filter scale. The final filter orientations are obtained by recalculating the $3 D R T$ for the ridge strength image $3 D R S_{\gamma}$, obtained using the optimized filter scale and initial filter orientation, and selecting the maximum value of the $3 D R T$. Finally, angular bandwidth $\left(\sigma_{\alpha}\right)$ is determined to be the peak kurtosis value of the Radon transform of the ridge strength image over multiple angular bandwidth values because a greater kurtosis indicates the variance is due to infrequent high-intensity voxel values. The final bone surface that was used during the registration algorithm is determined as the maximum of the 3D PS value; calculated using optimized filter parameters, along the direction of the US probe. 


\subsection{Point Cloud Simplification Using Particle Simulation}

In this work we employ a modification of the particle simulations (PSim) method developed by Pauly et al. [10] to decrease the number of points. The first step in PSim is to randomly select $n$ points from the original point cloud, $P_{S}$, resulting in a smaller subset of points denoted as $P_{s}$. Each point, $p_{i}$, in this subset is moved along the force $F_{t}=F_{i n}+F_{e x}+F_{j}$ and projected back onto the bone surface extracted in Section 2.2finding the nearest neighbor in $P_{S}$ [10]. $F_{i n}$ is the internal force of each point in $P_{s}$ acting on $p_{i}(4)$.

$$
F_{\text {in }}=\sum_{i=1}^{n} k\left(d-\left\|p-p_{i}\right\|\right) \times\left(\frac{p-p_{i}}{\left\|p-p_{i}\right\|}\right)
$$

Here $d$ defines the size of the neighborhood used to calculate force calculations. PSim was originally proposed for closed surfaces [10]; however the bone surfaces have boundaries at the edges of the data. In order to avoid $F_{\text {in }}$ forcing points towards low density areas, i.e. the edge of the surface, we have added $F_{e x}$, implemented as a trough function, which acts as a force to push points away from the edge. Finally, to ensure that points do not overlap we included a small perturbance $F_{j}$. After the force has been calculated, each point is moved according to $F_{t}$. This step is called the relaxation of the point cloud as described by Pauly et al [10]. These steps are iteratively repeated until equilibrium is reached. The force calculation above was implemented by Pauly et al [10] with an $O\left(n^{2}\right)$ complexity. As this calculation must be computed multiple times in the algorithm, it significantly adds to the overall runtime. We instead optimize the force calculations using the Barnes-Hut algorithm [11], modified to estimate $F_{t}$. The Barnes-Hut algorithm first models the point cloud as a hierarchical tree of; where each child node contains no more than one point. The tree is divided into octnode volumes, which are subdivisions of the parent node into eight equal parts. This tree is then used to estimate the distance of clusters of points from the point of interest; if points are sufficiently far away (determined by a constant, $\theta$, and the size of the octnode), the cluster can be modeled as a single point. This algorithm has a complexity of $O(n \log n)$ and reduces the runtime by approximately $50 \%$.

\subsection{Experimental Setup and Data Acquisition}

Our main clinical interest is to assess pelvis fractures, which include approximately $3 \%$ of all skeletal injuries [12]. Over $40 \%$ of patients requiring pelvic stabilization suffer longterm complications, usually neurological, urological and non-specific pain [12]. Therefore, the acute management of pelvic ring injuries is complex and demands precise surgery.

Phantom Study: We designed a phantom consisting of a high-resolution radioopaque Sawbone hemi-pelvis. The iliac crest region of the sawbone pelvis was cut and placed inside an open topped acrylic plastic cylindrical tube. The phantom was suspended in a PVC gel with sixty-one $1 \mathrm{~mm}$ metal fiducials suspended approximately 1-3 cm from the surface of the gel to ensure any US volume will contain between four and six fiducials (Fig.1.). This setup was scanned using a commercially available ultrasound machine (G.E. Voluson 730, GE Healthcare, Waukesha, WI) using a 3D RSP5-12 transducer. 49 US scans were acquired along the iliac spine of the phantom. The US volumes were $152 \times 198 \times 148$ voxels with an isotropic resolution of $0.25 \mathrm{~mm}$. 
The gold standard surface was provided by a high resolution CT machine (HRpQCT Xtreme CT, Scanco Medical, Wayne, Pennsylvania). The acquired CT volume size was a $482 \mathrm{~mm} \times 482 \mathrm{~mm} \times 402 \mathrm{~mm}$ volume with a resolution of $0.25 \mathrm{~mm}$. The fiducials were omitted from registration and were only used for validation purposes.

Clinical Study: The objective of the clinical study was to determine in a live operating room setting whether or not the proposed method could achieve successful registration results with the required accuracy. After obtaining all required ethics approvals, we obtained both CT and US scans from 21 consenting patients admitted to a Level 1 Trauma Centre with pelvic fractures that required a CT scan. The US scans were collected from the iliac crest regions. The voxel resolution for the CT volumes varied between $0.76 \mathrm{~mm}-0.83 \mathrm{~mm}$ in the $x$ and y axes and $1 \mathrm{~mm}-2 \mathrm{~mm}$ in the $z$ axis. We also provide registration accuracy comparison results where local phase features were obtained using empirical $v s$ optimized 3D Log-Gabor filter parameters.

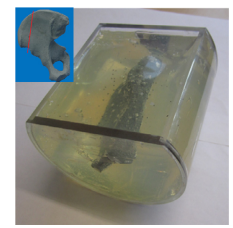

(a)

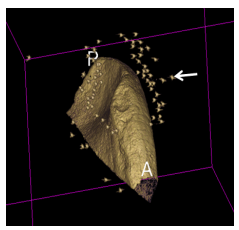

(b)

Fig. 1. Phantom validation experiment. (a) Constructed phantom comprised an iliac crest sawbone inside an open-topped acrylic plastic cylindrical tube filled with PVC (cutting plane illustrated in the top left corner). (b) High resolution CT scan of the constructed phantom (white arrow pointing to one of the fiducials used).

\section{Results and Discussion}

The GMM registration algorithm and PSim was implemented in $\mathrm{C}++$, whereas the curvature calculation and optimized 3DPS based bone surface extraction were implemented in MATLAB. All experiments were run on a $2.67 \mathrm{GHz}$ Intel(R) Core ${ }^{\mathrm{TM}} \mathrm{i}$ central processing unit (CPU) using 64-bit Windows 7 and a 4GB of RAM. The quality of the registration algorithm was evaluated by computing the root mean square error distance between the two registered surface representations, which we will denote as the surface registration error (SRE). We calculated target registration error (TRE) as the average distance between all corresponding fiducial pairs found in both volumes to validate the anatomical accuracy of our proposed method. The number of randomly selected points $(n)$ for PSim calculation was chosen as $n=500$ to provide the optimal trade-off between speed and accuracy. The results of the phantom setup showed an average $0.77 \mathrm{~mm}$ (SD $0.29 \mathrm{~mm}$ ) TRE with maximum TRE of $1.60 \mathrm{~mm}$ using the PSim point simplification method. The mean SRE for the phantom study using the proposed method was calculated as $0.31 \mathrm{~mm}$ (SD $0.09 \mathrm{~mm}$ ). For the naïve down sampling approach [6] the mean TRE increased to $6.84 \mathrm{~mm}$ (SD $5.35 \mathrm{~mm}$ ) with a maximum TRE value of $25.05 \mathrm{~mm}$. The average SRE for naïve down sampling method was $0.28 \mathrm{~mm}$ (SD 0.07) (Table 1.). The average run times for PSim and naïve down sampling method were $1.40 \mathrm{~s}(\mathrm{SD} 0.30 \mathrm{~s}$ ) and $1.01 \mathrm{~s}$ (SD $0.48 \mathrm{~s})$ respectively. The 
registration time decreased to $0.97 \mathrm{~s}$ (SD $0.55 \mathrm{~s}$ ) when $n$ was set to 300 . However, the SRE increased to $0.55 \mathrm{~mm}$ (SD $0.24 \mathrm{~mm}$ ), indicating using 500 points is more robust.

There were no fiducials available for the clinical validation; therefore, only the SRE was measured. The proposed method was able to register all 21 (100\%) clinical scans with a mean SRE of $0.72 \mathrm{~mm}$ (SD $0.66 \mathrm{~mm}$ ), while the naïve downsampling approach [6] was able to register only 10 scans (48\%). The mean SRE for the naïve downsampling approach was over $3 \mathrm{X}$ higher at $2.48 \mathrm{~mm}$ (SD $1.66 \mathrm{~mm}$ ).

Table 1. Quantitative results comparing registration using PSim vs naive downsampling for phantom and clinical scans

\begin{tabular}{|c|c|c|c|c||c|c|c|}
\hline \multirow{2}{*}{} & \multicolumn{4}{|c||}{ Phantom } & \multicolumn{3}{c|}{ Clinical } \\
\cline { 2 - 8 } & \multicolumn{2}{|c|}{ PSim } & \multicolumn{2}{c|}{ Naïve [6] } & PSim & Naïve [6] & Empirical PS \\
\cline { 2 - 8 } & $\begin{array}{c}\text { SRE } \\
(\mathrm{mm})\end{array}$ & $\begin{array}{c}\text { TRE } \\
(\mathrm{mm})\end{array}$ & $\begin{array}{c}\text { SRE } \\
(\mathrm{mm})\end{array}$ & $\begin{array}{c}\text { TRE } \\
(\mathrm{mm})\end{array}$ & $\begin{array}{c}\text { SRE } \\
(\mathrm{mm})\end{array}$ & $\begin{array}{c}\text { SRE } \\
(\mathrm{mm})\end{array}$ & $\begin{array}{c}\text { SRE } \\
(\mathrm{mm})\end{array}$ \\
\hline Mean & 0.31 & 0.77 & 0.28 & 6.84 & 0.72 & 2.48 & 1.88 \\
\hline SD & 0.09 & 0.29 & 0.07 & 5.35 & 0.66 & 1.66 & 1.75 \\
\hline Median & 0.29 & 0.79 & 0.27 & 5.76 & 0.66 & 0.92 & 4 \\
\hline Min & 0.15 & 0.25 & 0.18 & 0.80 & 0.01 & 0.08 & 0.32 \\
\hline Max & 0.65 & 1.60 & 0.48 & 25.05 & 2.25 & 4 & 4 \\
\hline
\end{tabular}

The success of registration is also affected by the extracted local phase features. Only 12 clinical scans (57\%) were successfully registered when using empirically determined filter parameters [7]. The mean SRE for this case was $1.88 \mathrm{~mm}$ (SD 1.75 $\mathrm{mm}$ ). It should be noted that the SRE was lower for all cases, as it is calculated from the extracted outlines which are determined from the 3DPS. Qualitative results of the clinical study can be seen in Figure 2. The US volumes were acquired in a region of the iliac spine unaffected by the fracture. The optimized filter parameters result in the extraction of sharper and more continuous surfaces (Fig. 2c) when compared to empirical method [7] (Fig. 2b). Investigating the registration result we can see that the proposed method successfully aligns the two volumes (Fig. 2d) where a close match between the surfaces is visible compared to the previous method (Fig. 2e).

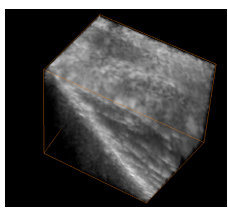

(a)

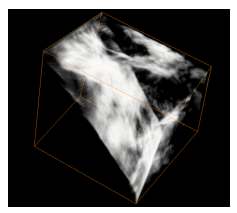

(b)

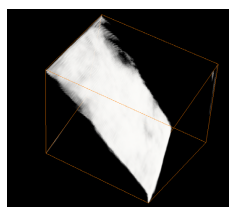

(c)

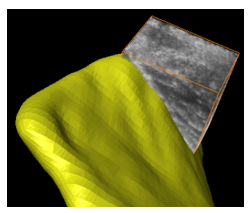

(d)

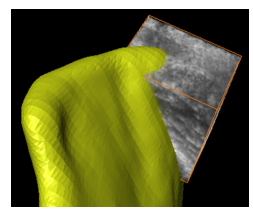

(e)

Fig. 2. Qualitative results on clinical data. (a) B-mode US volume obtained by scanning a patient's iliac crest. (b) 3D PS bone surface using empirical filter parameters. (c) Bone surface using our optimized filter parameters. (d) Registration using our proposed method. (e) Registration using naïve down sampling with empirical filter parameters. In (d) and (e) yellow surface is the ROI surface from the iliac crest region.

\section{Conclusions}

Accurate, robust and fast image registration is essential in US-based image guided interventions in order to provide real-time guidance. The previously proposed methods were either not robust enough to typical US artifacts or could not achieve the 
required speed or accuracy to be used in a live operating room setting. We presented a novel method for near real-time 3D US-CT registration in bone imaging deploying a statistical point-based registration in combination with a point cloud optimization method (PSim) that better represents the surface. We reduced the run-time by a further $50 \%$ by optimizing the force calculations used during point simplification. Furthermore, we incorporated a novel data driven selection of 3D log-Gabor filter parameters in the context of local phase based bone feature extraction in 3D US. The improvements achieved in terms of registration accuracy and robustness compared to the previously proposed state-of-the-art method were up to $89 \%$ and $52 \%$ for the phantom and clinical data, respectively [6]. Our future work will focus on optimizing the algorithms proposed in this paper to run on a GPU to achieve essentially real-time registration results and on validating our findings with a cadaver study.

\section{References}

1. Penney, G.P., Edwards, P.J., King, A.P., Blackall, J.M., Batchelor, P.G., Hawkes, D.J.: A Stochastic Iterative Closest Point Algorithm (stochastICP). In: Niessen, W.J., Viergever, M.A. (eds.) MICCAI 2001. LNCS, vol. 2208, pp. 762-769. Springer, Heidelberg (2001)

2. Moghari, M.H., Abolmaesumi, P.: Point-Based Rigid-Body Registration Using an Unscented Kalman Filter. IEEE Transactions on Medical Imaging 26(12), 1708-1728 (2007)

3. Penney, G., Barratt, D., Chan, C., Slomczykowski, M., Carter, T., Edwards, P., Hawkes, D.: Cadaver Validation of Intensity-Based Ultrasound to CT Registration. Medical Image Analysis 10(3), 385-395 (2006)

4. Gill, S., Abolmaesumi, P., Fichtinger, G., Boisvert, J., Pichora, D., Borshneck, D., Mousavi, P.: Biomechanically Constrained Groupwise Ultrasound to CT Registration of the Lumbar Spine. Medical Image Analysis (2010) (in press)

5. Khallaghi, S., Mousavi, P., Borschneck, D., Fichtinger, G., Abolmaesumi, P.: Biomechanically Constrained Groupwise Statistical Shape Model to Ultrasound Registration of the Lumbar Spine. In: Taylor, R.H., Yang, G.-Z. (eds.) IPCAI 2011. LNCS, vol. 6689, pp. 4754. Springer, Heidelberg (2011)

6. Brounstein, A., Hacihaliloglu, I., Guy, P., Hodgson, A., Abugharbieh, R.: Towards RealTime 3D US to CT Bone Image Registration Using Phase and Curvature Feature Based GMM Matching. In: Fichtinger, G., Martel, A., Peters, T. (eds.) MICCAI 2011, Part I. LNCS, vol. 6891, pp. 235-242. Springer, Heidelberg (2011)

7. Hacihaliloglu, I., Abugharbieh, R., Hodgson, A.J., Rohling, R.: Bone Segmentation and Fracture Detection in Ultrasound Using 3D Local Phase Features. In: Metaxas, D., Axel, L., Fichtinger, G., Székely, G. (eds.) MICCAI 2008, Part I. LNCS, vol. 5241, pp. 287-295. Springer, Heidelberg (2008)

8. Jian, B., Vemuri, B.: Robust Point Set Registration Using Gaussian Mixture Models. IEEE Transactions on Pattern Analysis and Machine Intelligence 33(8), 1633-1645 (2011)

9. Hacihaliloglu, I., Abugharbieh, R., Hodgson, A., Rohling, R.: Automatic Adaptive Parameterization in Local Phase Feature-Based Bone Segmentation in Ultrasound. Ultrasound in Med. and Biol. 37(10), 1689-1703 (2011)

10. Pauly, M., Gross, M., Kobbelt, L.: Efficient simplification of point- sampled surfaces. In: IEEE Proc. of Visualization (VIS 2002), pp. 163-170 (2002)

11. Barnes, J., Hut, P.: A hierarchical $\mathrm{O}(\mathrm{N} \log \mathrm{N})$ force-calculation algorithm. Nature 324 , 446-449 (1986)

12. Petrisora, B., Bhandarib, M.: Injuries to the pelvic ring: Incidence, classification, associated injuries and mortality rates. Current Orthopaedics 19(10), 327-333 (2005) 